\title{
EFFECTIVE BERTINI THEOREM AND FORMULAS FOR MULTIPLICITY AND THE LOCAL ŁOJASIEWICZ EXPONENT
}

\author{
TOMASZ RODAK, ADAM RÓŻYCKI, STANISŁAW SPODZIEJA
}

\begin{abstract}
The classical Bertini theorem on generic intersection of an algebraic set with hyperplanes states the following: Let $X$ be a nonsingular closed subvariety of $\mathbb{P}_{k}^{n}$, where $k$ is an algebraically closed field. Then there exists a hyperplane $H \subset \mathbb{P}_{k}^{n}$ not containing $X$ and such that the scheme $H \cap X$ is regular at every point. Furthermore, the set of hyperplanes with this property forms an open dense subset of the complete linear system $|H|$ considered as a projective space. We will show that one can effectively indicate a finite family of hyperplanes $H$ such that at least one of them satisfies the assertion of the Bertini theorem. As an application of the method used in the proof we will give effective formulas for the multiplicity and the Łojasiewicz exponent of polynomial mappings.
\end{abstract}

\section{INTRODUCTION}

The classical Bertini theorem on generic intersection of an algebraic set with hyperplanes is as follows (see [7, Theorem II.8.18]):

Bertini THEOREM 1. Let $X$ be a nonsingular closed subvariety of $\mathbb{P}_{k}^{m}$, where $k$ is an algebraically closed field. Then there exists a hyperplane $H \subset \mathbb{P}_{k}^{m}$ not containing $X$ and such that the scheme $H \cap X$ is regular at every point. (In fact, if $\operatorname{dim} X \geqslant 2$, then $H \cap X$ is connected, hence irreducible, and so $H \cap X$ is a nonsingular variety.) Furthermore, the set of hyperplanes with this property forms an open dense subset of the complete linear system $|H|$ considered as a projective space.

This theorem is one of the fundamental tools in algebraic geometry. For more details we refer to [8].

We will show that one can effectively indicate a finite family of hyperplanes $H$ such that at least one of them satisfies the assertion of the Bertini theorem (see Theorems 3.4, 3.6 and 3.8). Theorems 3.4 and 3.8 are formulated in terms of algebraic cones, i.e., algebraic sets defined by homogeneous polynomials. The

Date: June 22, 2021.

2010 Mathematics Subject Classification. 32B10, 14Q99, 12 Y99.

Key words and phrases. transversal intersection, multiplicity, Łojasiewicz exponent, finite mapping, effective formulas. 
most general is Theorem 3.8 which says (in the projective case) the following: Let $d, m, q, s$ be positive integers such that $m \geqslant q \geqslant s$, let

$$
\ell=d^{m-q}[(m-q)(d-1)+q]+m q-1,
$$

and let $N_{j}: \mathbb{C}^{(m+1) q} \rightarrow \mathbb{C}, 1 \leqslant j \leqslant \ell$, be a system of independent linear functions, i.e., any $(m+1) q$ of these functions are linearly independent. Then

$$
\begin{gathered}
K_{s, j_{1}, \ldots, j_{(m+1) q-1}}=\left\{x \in \mathbb{P}^{m}: \exists_{a=\left(a_{1}, \ldots, a_{q}\right) \in\left(\mathbb{P}^{m}\right)^{q}} N_{j_{1}}(a)=\cdots=N_{j_{(m+1) q-1}}(a)=0,\right. \\
\left.a_{1} x^{T}=\cdots=a_{s} x^{T}=0\right\} \quad \text { for } 1 \leqslant j_{1}<\cdots<j_{(m+1) q-1} \leqslant \ell
\end{gathered}
$$

is a system of linear subspaces of dimension $m-s$ such that for any irreducible and smooth algebraic set $V \subset \mathbb{P}^{m}$ of dimension $q$ and degree $d$, there are $1 \leqslant j_{1}<\cdots<$ $j_{(m+1) q-1} \leqslant \ell$ such that the intersection $X=V \cap K_{s, j_{1}, \ldots, j_{(m+1) q-1}}$ is transversal at any point $x \in X$ and the set $X$ is smooth. If moreover $q \geqslant s+1$ then $X$ is irreducible.

As a consequence of the Bertini theorem we obtain Corollary 3.11 which says that for any algebraic set $V=V\left(f_{1}, \ldots, f_{r}\right) \subset \mathbb{C}^{m}$ of pure dimension $q$, where $f_{j} \in \mathbb{C}[x], \operatorname{deg} f_{j} \leqslant d$ for $1 \leqslant j \leqslant r$, we have

$$
\operatorname{deg}_{0} V=\max _{1 \leqslant j_{1}<\cdots<j_{m(q-1)-1} \leqslant \ell} \operatorname{deg}_{0}\left(V \cap K_{s, j_{1}, \ldots, j_{m(q-1)-1}}\right) \quad \text { for any } 1 \leqslant s \leqslant q .
$$

A crucial role in the proof of the Bertini theorem is played by the following observation (see Lemma 2.3): Let $m, q, d$ be positive integers with $m \geqslant 2, m \geqslant q$, let $\ell=d(m-q)+q$, and let $N_{j}: \mathbb{C}^{m} \rightarrow \mathbb{C}, 1 \leqslant j \leqslant \ell$, be a system of independent linear functions. Then for any algebraic cone $C_{0} \subset \mathbb{C}^{m}$ with $\operatorname{dim} C_{0} \leqslant q$ and with total degree $\delta\left(C_{0}\right) \leqslant d$, there are $1 \leqslant i_{1}<\cdots<i_{q} \leqslant \ell$ such that

$$
C_{0} \cap V\left(N_{i_{1}}, \ldots, N_{i_{q}}\right)=\{0\} .
$$

The number $\ell$ in the above observation is optimal in terms of $d, m$ and $q$ (see Remark 2.4).

There are a lot of numerical invariants that could be associated with a polynomial map $f$. In this note we are interested in two of them, namely the multiplicity and the Łojasiewicz exponent. The multiplicity is a basic biholomorphic invariant characterizing the singularity of $f$ at zero (see [1, 4, 15, 16, 20]). As a corollary from the above observation we will prove the following effective formula for the multiplicity $i_{0}(f)$ of a finite polynomial mapping $f$ (see Theorem 4.1): Let $\ell=$ $d^{n}(m-n)+n$. For any independent system $L_{j}: \mathbb{C}^{m} \rightarrow \mathbb{C}, 1 \leqslant j \leqslant \ell$, and any polynomial mapping $f: \mathbb{C}^{n} \rightarrow \mathbb{C}^{m}$ of degree $d$, finite at 0 , we have

$$
i_{0}(f)=\min _{1 \leqslant i_{1}<\cdots<i_{n} \leqslant \ell} \operatorname{dim}_{\mathbb{C}} \mathcal{O} /\left(L_{i_{1}} \circ f, \ldots, L_{i_{n}} \circ f\right) .
$$

Note that in the case $m>n$, we only have $i_{0}(f) \geqslant \operatorname{dim}_{\mathbb{C}} \mathcal{O} /\left(f_{1}, \ldots, f_{m}\right)$. 
For the Łojasiewicz exponent (see [13, 14, 10, 11, the definition is given in Section 5), we obtain the following formulas (see Corollaries 5.3 and 5.6): Let $m \geqslant n$ be positive integers, let $\ell=d^{n}(m-n)+n$, and let $L_{j}: \mathbb{C}^{m} \rightarrow \mathbb{C}, 1 \leqslant j \leqslant \ell$, be a system of independent linear functions. Then for any polynomial mapping $f:\left(\mathbb{C}^{n}, 0\right) \rightarrow\left(\mathbb{C}^{m}, 0\right)$ finite at 0 with $\operatorname{deg} f \leqslant d$ we have

$$
\mathcal{L}_{0}(f)=\min _{1 \leqslant i_{1}<\cdots<i_{n} \leqslant \ell} \mathcal{L}_{0}\left(\left(L_{i_{1}}, \ldots, L_{i_{n}}\right) \circ f\right)=\min _{1 \leqslant i_{1}<\cdots<i_{n} \leqslant \ell} \mathcal{L}_{0}\left(H_{f,\left(L_{i_{1}}, \ldots, L_{i_{n}}\right)}\right),
$$

where $H_{f, L}(z)=L \circ f(z)+\left(z_{1}^{d^{n}+1}, \ldots, z_{n}^{d^{n}+1}\right), z=\left(z_{1}, \ldots, z_{n}\right) \in \mathbb{C}^{n}$ for a linear mapping $L: \mathbb{C}^{m} \rightarrow \mathbb{C}^{n}$. Then by using Płoski's formula for the Łojasiewicz exponent of mappings $\mathbb{C}^{n} \rightarrow \mathbb{C}^{n}$ (see [18, 19]) we obtain an effective formula for the exponent in terms of orders of polynomials $P_{f, L, N} \in \mathbb{C}\left[y_{1}, \ldots, y_{n}, t\right]$ defining the sets of values of the mappings

$$
\Phi_{f, L, N}(z)=\left(H_{f, L}(z), N(z)\right),
$$

where $N: \mathbb{C}^{n} \rightarrow \mathbb{C}$ is a linear function. More precisely, we obtain the following formula (see Theorem 5.7): Let $\ell_{L}=d^{n}(m-n)+n, \ell_{N}=n+\left[\left(d^{n}+1\right)^{n}-1\right] n(n-1)$, and let $\mathbb{I}_{L}=\left\{\mathbf{s}=\left(s_{1}, \ldots, s_{n}\right) \in \mathbb{N}^{n}: 1 \leqslant s_{1}<\cdots<s_{n} \leqslant \ell_{L}\right\}$. Then for any sequences $N_{i}: \mathbb{C}^{n} \rightarrow \mathbb{C}, 1 \leqslant i \leqslant \ell_{N}, L_{j}: \mathbb{C}^{m} \rightarrow \mathbb{C}, 1 \leqslant j \leqslant \ell_{L}$, of independent linear functions we have

$$
\mathcal{L}_{0}(f)=\min _{\mathbf{s} \in \mathbb{I}_{L}} \max _{1 \leqslant i \leqslant \ell_{N}} \frac{1}{\Delta\left(P_{f, L_{\mathbf{s}}, N_{i}}\right)}
$$

where

$$
\Delta(P)=\min _{j=0}^{r} \frac{\operatorname{ord}_{y} P_{j}}{r+1-j}
$$

if $P(y, t)=\sum_{j=0}^{\infty} P_{j}(y) t^{j} \in \mathbb{C}\{y, t\}$ is a power series regular of order $r+1$ in $t$.

In [19, Theorem 7] we obtained a similar result to Theorem 5.7. There, however, we used the linear mappings $L$ and $N$ with generic coefficients. An important limitation in using [19, Theorem 7] is a quite large number, $n(m+1)$, of additional variables (i.e., coefficients of $L$ and $N$ ) needed to determine the polynomial $P_{f, L, N}$. This results in an exponential extension of the time needed for calculations. Theorem 5.7 provides a formula for the Lojasiewicz exponent without using additional variables. However, we need to repeat the calculations $\ell_{N}\left(\begin{array}{c}\ell_{L} \\ n\end{array}\right)$ times. From the point of view of the formula's effectiveness and algorithmic implementation, this is a significant simplification and reduces the time of computer calculations considerably.

\section{Preliminaries}

1.1. Basic notations. By $f:\left(\mathbb{C}^{m}, 0\right) \rightarrow\left(\mathbb{C}^{n}, 0\right)$ we denote a mapping defined in a neighbourhood of $0 \in \mathbb{C}^{m}$ with values in $\mathbb{C}^{n}$ such that $f(0)=0$. A holomorphic mapping $f:\left(\mathbb{C}^{m}, 0\right) \rightarrow\left(\mathbb{C}^{n}, 0\right)$ is called finite at 0 if 0 is an isolated point of $f^{-1}(0)$. 
We denote by $\mathbb{C}[x]$ the ring of complex polynomials in $x=\left(x_{1}, \ldots, x_{m}\right)$. The degree of a polynomial $f \in \mathbb{C}[x]$ is denoted by $\operatorname{deg} f$. We put $\operatorname{deg} 0=-\infty$. Let $f=\left(f_{1}, \ldots, f_{r}\right): \mathbb{C}^{m} \rightarrow \mathbb{C}^{r}$ be a polynomial mapping, i.e., $f_{j} \in \mathbb{C}[x]$ for $1 \leqslant j \leqslant r$. By the degree of $f$, denoted by $\operatorname{deg} f$, we mean $\max \left\{\operatorname{deg} f_{j}: 1 \leqslant j \leqslant r\right\}$.

For any set $\mathcal{I} \subset \mathbb{C}[x]$, we denote by $V(\mathcal{I}) \subset \mathbb{C}^{m}$ the set of common zeros of all polynomials $f \in \mathcal{I}$; any set of the form $V(\mathcal{I})$ is called algebraic. We put $V(\emptyset)=\mathbb{C}^{m}$. By the Hilbert basis theorem $V(\mathcal{I})=V(\mathcal{J})=V\left(f_{1}, \ldots, f_{r}\right)$ for some polynomials $f_{j} \in \mathbb{C}[x], 1 \leqslant j \leqslant r$, belonging to the ideal $\mathcal{J} \subset \mathbb{C}[x]$ generated by $\mathcal{I}$. If the polynomials $f_{j}, 1 \leqslant j \leqslant r$, are homogeneous, we call the set $V\left(f_{1}, \ldots, f_{r}\right)$ an algebraic cone.

We denote by $\operatorname{dim} V$ the dimension of an algebraic (or locally analytic) set $V \subset$ $\mathbb{C}^{n}$. We set $\operatorname{dim} \emptyset=-1$. Let $\operatorname{dim}_{a} V$ denote the dimension of $V$ at $a \in \mathbb{C}^{n}$, i.e., the dimension of the germ of the set $V$ at $a$. The set $V$ is called pure dimensional if all irreducible components of $V$ have the same dimension.

1.2. Tangent cone. Let $C_{0}(V)$ be the tangent cone to a set $V \subset \mathbb{C}^{m}$ at 0 in the sense of Whitney [25], i.e., the set of vectors $w \in \mathbb{C}^{m}$ for which there are sequences $p_{\nu} \in V$ with $p_{\nu} \rightarrow 0$ and $\alpha_{\nu} \in \mathbb{C}$ such that $\alpha_{\nu} p_{\nu} \rightarrow w$ as $\nu \rightarrow \infty$. We have the following fact (cf. [25, Theorem 8.10 and Lemma 8.11]).

FACT 1.1. Let $V \subset \mathbb{C}^{m}$ be an analytic set in a neighbourhood of the origin with $0 \in V$. Then $C_{0}(V)$ is an algebraic cone and $\operatorname{dim} C_{0}(V)=\operatorname{dim}_{0} V$. If 0 is a simple point of $V$ then $C_{0}(V)$ is the tangent space $T_{0}(V)$ to $V$ at 0 .

1.3. Degree of algebraic sets. The degree of a pure dimensional algebraic set $V \subset \mathbb{C}^{m}$, denoted by $\operatorname{deg} V$, is defined as the number of common points of $V$ and a generic affine subspace $X \subset \mathbb{C}^{m}$ of dimension $m-\operatorname{dim} V$; and the degree of $V$ at a point $a \in V$, denoted by $\operatorname{deg}_{a} V$, is the multiplicity $i_{a}(V, X)$ of proper intersection of $V$ with $X$ as above such that $a \in X$ (see [3]). Obviously, $\operatorname{deg}_{a} V \leqslant \operatorname{deg} V$.

Let $V=V_{1} \cup \cdots \cup V_{s}$ be the decomposition of an algebraic set $V \subset \mathbb{C}^{m}$ into irreducible components. The number

$$
\delta(V):=\operatorname{deg} V_{1}+\cdots+\operatorname{deg} V_{s}
$$

is called the total degree of $V$ (see [15).

We have the following useful properties of the total degree (see [15]).

FACT 1.2. If $V, W \subset \mathbb{C}^{m}$ are algebraic sets, then

$$
\begin{aligned}
& \delta(V \cap W) \leqslant \delta(V) \delta(W), \\
& \delta(V) \leqslant \operatorname{deg} f_{1} \cdots \operatorname{deg} f_{r}, \quad \text { where } V=V\left(f_{1}, \ldots, f_{r}\right), \\
& \delta(\overline{L(V)}) \leqslant \delta(V) \quad \text { for any linear mapping } L: \mathbb{C}^{m} \rightarrow \mathbb{C}^{k} .
\end{aligned}
$$


FACT 1.3. If $V \subset \mathbb{C}^{m}$ is an algebraic set, then $\delta\left(C_{0}(V)\right) \leqslant \delta(V)$. If $f: \mathbb{C}^{n} \rightarrow \mathbb{C}^{m}$ is a polynomial mapping of degree $d$, finite at 0 , then $\delta\left(C_{0}\left(\overline{f\left(\mathbb{C}^{n}\right)}\right)\right) \leqslant d^{n}$.

Proof. The first assertion immediately follows from [3, Theorem 6.4]. We will prove the second assertion. Let $W=f\left(\mathbb{C}^{n}\right), k=\operatorname{dim} \bar{W}$ and $D=\operatorname{deg} \bar{W}$. Then $k \leqslant n$ and by the definition of the degree of an algebraic set and the fact that $\operatorname{dim} \overline{\bar{W} \backslash W}<k$, for a generic linear mapping $L: \mathbb{C}^{m} \rightarrow \mathbb{C}^{k}$ and a generic $z \in \mathbb{C}^{k}, \# L^{-1}(z) \cap W=D$, where \# stands for cardinality. Hence $D \leqslant \delta\left((L \circ f)^{-1}(z)\right)$. Since for $y \in \mathbb{C}^{m}$ such that $L(y)=z$ we have $(L \circ f)^{-1}(z)=(L \circ(f-y))^{-1}(0)$, (2) gives $D \leqslant d^{k} \leqslant d^{n}$. Consequently, the first assertion implies the second.

We denote by $\mathbb{L}(m, r)$ the set of linear mappings $\mathbb{C}^{m} \rightarrow \mathbb{C}^{r}$, where $m, r \in \mathbb{N}$. We set $\mathbb{L}(m, r)=\{0\}$ if $r=0$.

We will need the following lemma (cf. [9, Lemma 3.20] and [21, Lemma 1.1]).

Lemma 1.4. Let $f=\left(f_{1}, \ldots, f_{r}\right): \mathbb{C}^{n} \rightarrow \mathbb{C}^{r}$ be a polynomial mapping such that $\operatorname{dim} V(f)=q$. Let $W$ be the union of the irreducible components of dimension $q$ of the set $V(f)$. Then for a generic linear mapping $L=\left(L_{1}, \ldots, L_{n-q}\right) \in \mathbb{L}(r, n-q)$ the set $V_{1}=V\left(L_{1} \circ f, \ldots, L_{n-q} \circ f\right)$ has dimension $q, W$ is the union of some irreducible components of $V_{1}$, and

$$
\operatorname{deg} W \leqslant \delta\left(V_{1}\right) \leqslant \operatorname{deg}\left(L_{1} \circ f\right) \cdots \operatorname{deg}\left(L_{n-q} \circ f\right) .
$$

Proof. Without loss of generality we may assume that $q \geqslant 0$. Let $Y=\overline{f\left(\mathbb{C}^{n}\right)} \subset \mathbb{C}^{r}$. By Chevalley's theorem (see [15, p. 395]) the set $Y$ is algebraic and obviously irreducible. Moreover, [16, Theorem 3.13] easily implies that $r \geqslant \operatorname{dim}_{0} Y=\operatorname{dim} Y \geqslant n-$ $q$, and there exists a Zariski open and dense subset $U$ of $Y$ such that $\operatorname{dim} f^{-1}(y) \leqslant q$ for $y \in U$ and obviously $\operatorname{dim}(Y \backslash U) \leqslant \operatorname{dim} Y-1$.

Let $k=\operatorname{dim} C_{0}(Y)$. By Fact 1.1, $k=\operatorname{dim} Y$ and so $r \geqslant k \geqslant n-q$.

If $k<r$, by definition of the degree of an algebraic set, for a generic linear mapping $L: \mathbb{C}^{r} \rightarrow \mathbb{C}^{k}$ the set $Y \cap L^{-1}(0)$ is finite, $(Y \backslash U) \cap L^{-1}(0) \subset\{0\}$ and obviously $0 \in Y \cap L^{-1}(0)$. So, $V(L \circ f)$ has dimension $q$ and contains $W$. Consequently, $W$ is the union of some irreducible components of $V(L \circ f)$. Hence, it suffices to consider the case $k=r$, and then obviously $Y=\mathbb{C}^{r}$ and $r \leqslant n$.

Let

$$
Z=\left\{(x, L) \in \mathbb{C}^{n} \times \mathbb{L}(r, n-q): L \circ f(x)=0\right\},
$$

and let

$$
\pi: Z \ni(x, L) \mapsto L \in \mathbb{L}(r, n-q) .
$$

Obviously $\operatorname{dim} \mathbb{L}(r, n-q)=r(n-q)$. Since $Y=\mathbb{C}^{r}$ and $r \leqslant n$, we have $\overline{\pi(Z)}=$ $\mathbb{L}(r, n-q), \operatorname{dim} Z=r(n-q)+q$ and for a generic $L \in \mathbb{L}(r, n-q)$ the set $\pi^{-1}(L)$ has 
dimension $q$. This gives the first assertion. The second follows immediately from the first and Fact 1.2

From Lemma 1.4 we immediately obtain

Corollary 1.5. If $V=V\left(f_{1}, \ldots, f_{r}\right) \subset \mathbb{C}^{n}$ is an algebraic cone of pure dimension $q$, where $f_{1}, \ldots, f_{r}: \mathbb{C}^{n} \rightarrow \mathbb{C}$ are nonzero homogeneous polynomials with $\operatorname{deg} f_{j} \leqslant d$ for $1 \leqslant j \leqslant r$, then

$$
\delta(V) \leqslant d^{n-q}
$$

1.4. Projective algebraic sets. Let $\mathbb{P}^{m}$ be the $m$-dimensional complex projective space. Let $V \subset \mathbb{P}^{m}$ be an algebraic set, i.e., the set of common zeros of some system $S \subset \mathbb{C}\left[x_{0}, \ldots, x_{m}\right]$ of homogeneous polynomials. Let $\mathcal{I} \subset \mathbb{C}\left[x_{0}, \ldots, x_{m}\right]$ be the radical of the ideal generated by $S$. Then $\mathcal{I}$ is the ideal of all polynomials vanishing on $V$ and

$$
V^{\mathbb{C}}:=\left\{\left(x_{0}, \ldots, x_{m}\right) \in \mathbb{C}^{m+1}:\left[x_{0}: \ldots: x_{m}\right] \in V\right\} \cup\{0\}=V(\mathcal{I}),
$$

where $V(\mathcal{I})=\left\{x \in \mathbb{C}^{m+1}: f(x)=0\right.$ for any $\left.f \in \mathcal{I}\right\}$, is an algebraic cone. We have $\operatorname{dim} V=\operatorname{dim} V^{\mathbb{C}}-1$. The set $V$ is called pure dimensional if all irreducible components of $V$ have the same dimension.

Since any hyperplane $H \subset \mathbb{C}^{m+1}$ of dimension $k$ can be written in the form $V\left(L_{1}, \ldots, L_{m-k}, L_{m-k+1}-a\right)$ for some $L_{1}, \ldots, L_{m-k+1} \in \mathbb{L}(m, 1)$ and $a \in \mathbb{C}$, we can define the degree of $V$ by $\operatorname{deg} V=\operatorname{deg} V^{\mathbb{C}}$ (if $V$ is of pure dimension), and its total degree by $\delta(V)=\delta\left(V^{\mathbb{C}}\right)$.

\section{Auxiliary Results}

2.1. Main lemma. A system of functions $N_{1}, \ldots, N_{s} \in \mathbb{L}(m, 1), s \geqslant m$, will be called independent if for any sequence $1 \leqslant i_{1}<\cdots<i_{m} \leqslant s$ the system $N_{i_{1}}, \ldots, N_{i_{m}}$ is linearly independent over $\mathbb{C}$.

Remark 2.1. Take a system $N_{1}, \ldots, N_{s} \in \mathbb{L}(m, 1)$ with $s \geqslant n$, and let $N_{i}(x)=$ $a_{i, 1} x_{1}+\cdots+a_{i, m} x_{m}$. Then the system $N_{1}, \ldots, N_{s}$ is independent if and only if $\prod_{1 \leqslant i_{1}<\cdots<i_{m} \leqslant s} \operatorname{det}\left[a_{i_{j}, l}\right]_{1 \leqslant j, l \leqslant m} \neq 0$. So, the set of independent systems is a dense subset of $\mathbb{L}(m, s)$ with algebraic complement.

EXAmple 2.2. For any injective sequence $a_{i} \in \mathbb{C}, 1 \leqslant i \leqslant s, s \geqslant m$, the system

$$
N_{i}\left(x_{1}, \ldots, x_{m}\right)=x_{1}+a_{i} x_{2}+\cdots+a_{i}^{m-1} x_{m}, \quad 1 \leqslant i \leqslant s,
$$

is independent. Indeed, this follows from the properties of the Vandermonde determinant.

A crucial role in the discussions below will be played by the following lemma. 
Lemma 2.3. Let $m, q, d$ be positive integers with $m \geqslant 2, m \geqslant q$, let $\ell=d(m-q)+q$, and let $N_{j} \in \mathbb{L}(m, 1), 1 \leqslant j \leqslant \ell$, be a system of independent linear functions. Then for any algebraic cone $C_{0} \subset \mathbb{C}^{m}$ with $\operatorname{dim} C_{0} \leqslant q$ and $\delta\left(C_{0}\right) \leqslant d$, there exist $1 \leqslant i_{1}<\cdots<i_{q} \leqslant \ell$ such that

$$
C_{0} \cap V\left(N_{i_{1}}, \ldots, N_{i_{q}}\right)=\{0\} .
$$

Proof. The proof is by induction on $q \in\{1, \ldots, m\}$. Take any algebraic cone $C_{0} \subset$ $\mathbb{C}^{m}$ with $\operatorname{dim} C_{0}=1$ and $\delta\left(C_{0}\right) \leqslant d$. Then there are $w_{1}, \ldots, w_{d} \in \mathbb{C}^{m} \backslash\{0\}$ such that $C_{0}=w_{1} \mathbb{C} \cup \cdots \cup w_{d} \mathbb{C}$. Suppose that for any $i \in\{1, \ldots, \ell\}$ there exists $\varphi(i) \in$ $\{1, \ldots, d\}$ such that $N_{i}\left(w_{\varphi(i)}\right)=0$. Since $w_{\varphi(i)} \neq 0$, the choice of $N_{1}, \ldots, N_{\ell}$ yields $\# \varphi^{-1}(j) \leqslant m-1$, so

$$
d(m-1)+1=\ell=\# \varphi^{-1}(\{1, \ldots, d\})=\# \varphi^{-1}(1)+\cdots+\# \varphi^{-1}(d) \leqslant d(m-1),
$$

which is impossible. This gives the assertion for $q=1$.

Assume that the assertion holds for $1 \leqslant q-1<m$. Take any algebraic cone $C_{0}$ with $\operatorname{dim} C_{0} \leqslant q$ and $\delta\left(C_{0}\right) \leqslant d$. If $q=m$ then the assertion is obvious. Assume that $q<m$. Let $C_{0}=V_{1} \cup \cdots \cup V_{s}$ be the decomposition into irreducible components. Since $\delta\left(C_{0}\right) \leqslant d$, we have $s \leqslant d$. Observe that there exists $i \in\{1, \ldots, \ell\}$, such that $\operatorname{dim} C_{0} \cap V\left(N_{i}\right)<q$. Indeed, suppose that for any $i \in\{1, \ldots, \ell\}$ there exists $\psi(i) \in\{1, \ldots, s\}$ such that $V_{\psi(i)} \subset V\left(N_{i}\right)$ and $\operatorname{dim} V_{\psi(i)}=q$. Analogously to the above, the choice of $N_{1}, \ldots, N_{\ell}$ yields $\# \psi^{-1}(j) \leqslant m-q$, so

$$
d(m-q)+q=\ell=\psi^{-1}(\{1, \ldots, s\}) \leqslant s(m-q) \leqslant d(m-q),
$$

which is impossible and gives the announced observation.

By the above observation we can take a function $N_{i}$ with $\operatorname{dim} C_{1} \leqslant q-1$ and $\delta\left(C_{1}\right) \leqslant d$, where $C_{1}=C_{0} \cap V\left(N_{i}\right)$. After a linear change of coordinates, we can assume that $N_{i}\left(x_{1}, \ldots, x_{m}\right)=x_{m}$. Then the family $\left.N_{j}\right|_{\mathbb{C}^{m-1} \times\{0\}}, j \neq i$, contains $d(m-q)+q-1=d((m-1)-(q-1))+q-1$ independent linear functions in $x_{1}, \ldots, x_{m-1}$. So, the induction hypothesis gives the assertion for $q$ and the proof is complete.

REMARK 2.4. The number $\ell=d(m-q)+q$ in Lemma 2.3 is optimal. Indeed, take an independent system of functions $N_{j} \in \mathbb{L}(m, 1), 1 \leqslant j \leqslant \ell$. Let

$$
V_{i}=V\left(N_{(i-1)(m-q)+1}, \ldots, N_{(i-1)(m-q)+m-q}\right), \quad i=1, \ldots, d .
$$

Then $C_{0}=V_{1} \cup \cdots \cup V_{d} \subset \mathbb{C}^{m}$ is an algebraic cone, $\operatorname{dim}\left(C_{0}\right)=q, \delta\left(C_{0}\right)=d$, and

$$
\begin{aligned}
& C_{0} \cap V\left(N_{i_{1}}, \ldots, N_{i_{q}}\right) \neq\{0\} \quad \text { for } 1 \leqslant i_{1}<\cdots<i_{q} \leqslant \ell \text { with } i_{1} \leqslant d(m-q), \\
& C_{0} \cap V\left(N_{d(m-q)+1}, \ldots, N_{d(m-q)+q}\right)=\{0\} .
\end{aligned}
$$


2.2. Corollaries from Lemma 2.3. From Lemma 2.3 we immediately obtain (see [3. Theorem 6.3])

Corollary 2.5. Let $d, m, q$ be positive integers such that $m \geqslant q$, let $\ell=d(m-$ $q)+q$, and let $N_{j} \in \mathbb{L}(m(q-1), 1), 1 \leqslant j \leqslant \ell$, be a system of independent linear functions. Then for any algebraic set $V=V\left(f_{1}, \ldots, f_{r}\right) \subset \mathbb{C}^{m}$ of pure dimension $q$, where $f_{j} \in \mathbb{C}[x], \operatorname{deg} f_{j} \leqslant d$ for $1 \leqslant j \leqslant r$, we have

$$
\operatorname{deg}_{0} V=\min _{1 \leqslant i_{1}<\cdots<i_{q} \leqslant \ell} i_{0}\left(V, V\left(N_{i_{1}}, \ldots, N_{i_{q}}\right)\right) .
$$

Corollary 2.6. Let $C_{0} \subset \mathbb{C}^{m}$ be an algebraic cone of pure dimension $q$ and degree $d$.

(a) If $N_{1}, \ldots, N_{q} \in \mathbb{L}(m, 1)$ is a system of linear functions such that $C_{0} \cap$ $V\left(N_{1}, \ldots, N_{q}\right)=\{0\}$, then for any $1 \leqslant i_{1}<\cdots<i_{s} \leqslant q$ the intersection $C_{0} \cap V\left(N_{i_{1}}, \ldots, N_{i_{s}}\right)$ is proper.

(b) For any system of independent linear functions $N_{j} \in \mathbb{L}(m, 1), 1 \leqslant j \leqslant \ell$, $\ell=d(m-q)+q$, where $d=\operatorname{deg} C_{0}$, there exist $1 \leqslant j_{1}<\cdots<j_{q} \leqslant \ell$ such that for any $1 \leqslant i_{1}<\cdots, i_{s} \leqslant q$ the intersection $C_{0} \cap V\left(N_{j_{i_{1}}}, \ldots, N_{j_{i_{s}}}\right)$ is proper.

Proof. Let $C_{1}$ be an irreducible component of $C_{0}$. Take any $1 \leqslant i_{1}<\cdots<i_{s} \leqslant q$ and let $\sigma:\{1, \ldots, q\} \rightarrow\{1, \ldots, q\}$ be a bijection such that $\sigma(j)=i_{j}$ for $1 \leqslant j \leqslant s$. By [16, Theorem 3.13] for any $1 \leqslant i \leqslant q$ we have

$$
\operatorname{dim}\left(C_{1} \cap V\left(N_{\sigma(1)}, \ldots, N_{\sigma(i-1)}\right)\right)-1 \leqslant \operatorname{dim}\left(C_{1} \cap V\left(N_{\sigma(1)}, \ldots, N_{\sigma(i)}\right)\right) .
$$

Since $\operatorname{dim} C_{1}=q$ and $\operatorname{dim}\left(C_{1} \cap V\left(N_{\sigma(1)}, \ldots, N_{\sigma(q)}\right)\right)=0$, for $1 \leqslant i \leqslant q$ we have

$$
\operatorname{dim}\left(C_{1} \cap V\left(N_{\sigma(1)}, \ldots, N_{\sigma(i-1)}\right)\right)-1=\operatorname{dim}\left(C_{1} \cap V\left(N_{\sigma(1)}, \ldots, N_{\sigma(i)}\right)\right),
$$

and consequently $\operatorname{dim}\left(C_{1} \cap V\left(N_{\sigma(1)}, \ldots, N_{\sigma(s)}\right)\right)=q-s$, which gives (a). From (a) and Lemma 2.3 we immediately obtain (b).

From Lemma 2.3 for $q=m-1$ we immediately obtain

Corollary 2.7. Let $m, d$ be positive integers with $m \geqslant 2$, let $\ell=d+m-1$, and let $N_{j} \in \mathbb{L}(m, 1), 1 \leqslant j \leqslant \ell$, be a system of independent linear functions. Then for any algebraic cone $C_{0} \subset \mathbb{C}^{m}$ with $\operatorname{dim} C_{0}<m$ and $\delta\left(C_{0}\right) \leqslant d$, there exist $1 \leqslant i_{1}<\cdots<i_{m-1} \leqslant \ell$ such that $V\left(N_{i_{1}}, \ldots, N_{i_{m-1}}\right) \cap C_{0} \subset\{0\}$. Moreover, the sets $V\left(N_{i_{1}}, \ldots, N_{i_{m-1}}\right), 1 \leqslant i_{1}<\cdots<i_{m-1} \leqslant \ell$, are one-dimensional linear subspaces of $\mathbb{C}^{m}$.

From Corollary 2.7 and Example 2.2 we immediately obtain 
Corollary 2.8. Let $N_{j}\left(x_{1}, \ldots, x_{m}\right)=x_{1}+a_{j} x_{2}+\cdots+a_{j}^{m-1} x_{m}$, where $a_{j} \in$ $\mathbb{C} \backslash\{0\}$ are pairwise different numbers, $1 \leqslant j \leqslant \ell, \ell=d+m-1, d>0$. Then for any $1 \leqslant j_{1}<\cdots<j_{m-1} \leqslant \ell$ there exists a unique point

$$
F\left(a_{j_{1}}, \ldots, a_{j_{m-1}}\right) \in V\left(N_{j_{1}}, \ldots, N_{j_{m-1}}\right)
$$

with first coordinate 1 . Moreover, for any algebraic cone $C \subset \mathbb{C}^{m}$ with $\operatorname{dim} C<m$ and $\delta(C) \leqslant d$ there exist $1 \leqslant j_{1}<\cdots<j_{m-1} \leqslant \ell$ such that $F\left(a_{j_{1}}, \ldots, a_{j_{m-1}}\right) \notin C$.

REMARK 2.9. Let $n=m-1>0$. By using the Lagrange interpolation formula we easily find that the function

$$
F=\left(F_{0}, \ldots, F_{n}\right): \mathbb{C}^{n} \ni\left(a_{1}, \ldots, a_{n}\right) \mapsto F\left(a_{1}, \ldots, a_{n}\right) \in \mathbb{C}^{n+1}
$$

in Corollary 2.8 is defined by $F_{0}\left(a_{1}, \ldots, a_{n}\right)=1$ and

$F_{k}\left(a_{1}, \ldots, a_{n}\right)=(-1)^{n-k+1} \sum_{j=1}^{n}\left(a_{j} \prod_{\substack{1 \leqslant i \leqslant m \\ i \neq j}}\left(a_{j}-a_{i}\right)\right)^{-1} \sum_{\substack{1 \leqslant i_{1}<\cdots<i_{n-k} \leqslant n \\ i_{s} \neq j}} a_{i_{1}} \cdots a_{i_{n-k}}$.

for $k=1, \ldots, n$, where $a_{i} \neq 0$ and $a_{i} \neq a_{j}$ for $i \neq j$.

We have the following generalization of Lemma 2.3 .

Corollary 2.10. Let $k, m, q, d$ be positive integers with $m \geqslant 2, m \geqslant q$ and $k \geqslant q$. Let

$$
\ell_{k}=k+d(m-1)\left(\begin{array}{l}
k \\
q
\end{array}\right)-d(q-1)
$$

and let $N_{j} \in \mathbb{L}(m, 1), 1 \leqslant j \leqslant \ell_{k}$ be a system of independent linear functions. Then for any algebraic cone $C_{0} \subset \mathbb{C}^{m}$ with $\operatorname{dim} C_{0} \leqslant q$ and $\delta\left(C_{0}\right) \leqslant d$, there exist $1 \leqslant j_{1}<\cdots<j_{k} \leqslant \ell_{k}$ such that for any $1 \leqslant i_{1}<\cdots<i_{q} \leqslant k$,

$$
C_{0} \cap V\left(N_{j_{i_{1}}}, \ldots, N_{j_{i_{q}}}\right)=\{0\} .
$$

Proof. The proof is by induction on $k \geqslant q$. If $k=q$ then the assertion follows from Lemma 2.3. Assume that the assertion holds for $k-1 \geqslant q$. Take an algebraic cone $C_{0} \subset \mathbb{C}^{m}$ with $\operatorname{dim} C_{0} \leqslant q$ and $\delta\left(C_{0}\right) \leqslant d$. By the induction hypothesis there are $1 \leqslant j_{1}<\cdots<j_{k-1} \leqslant \ell_{k-1}$ such that for any $1 \leqslant i_{1}<\cdots<i_{q} \leqslant k-1$ the equality (5) holds. Let

$$
C_{1}=\bigcup_{1 \leqslant i_{1}<\cdots<i_{q-1} \leqslant k-1}\left[C_{0} \cap V\left(N_{j_{i_{1}}}, \ldots, N_{j_{i_{q-1}}}\right)\right] .
$$

By (5) we see that $\operatorname{dim} C_{1} \leqslant 1$ and by (11) in Fact 1.2, $\delta\left(C_{1}\right) \leqslant\left(\begin{array}{c}k-1 \\ q-1\end{array}\right) d$. Since $\ell_{k}-\ell_{k-1}=\left(\begin{array}{c}k-1 \\ q-1\end{array}\right) d(m-1)+1$, by Lemma 2.3 there exists $j_{k} \in\left\{\ell_{k-1}+1, \ldots, \ell_{k}\right\}$ such that $C_{1} \cap V\left(N_{j_{k}}\right)=\{0\}$. So, for $j_{1}, \ldots, j_{k}$ we easily obtain the assertion and the proof is complete. 
REMARK 2.11. The number $\ell_{k}$ in Corollary 2.10 is optimal. Indeed, by Remark 2.4. $\ell_{q}=\ell$ is optimal for $k=q$. Assume that $\ell_{k-1}$ is optimal for $k-1 \geqslant q$. Then by arguing as in the proof of Corollary 2.10 we see that there exists an algebraic cone $C_{0} \subset \mathbb{C}^{n}$ of pure dimension $q$ with $\delta\left(C_{0}\right)=d$ such that the cone $C_{1}$ defined by (6) is of pure dimension 1 and $\delta\left(C_{1}\right)=\left(\begin{array}{c}k-1 \\ q-1\end{array}\right) d$. So, by Remark 2.4 we need an additional $\left(\begin{array}{l}k-1 \\ q-1\end{array}\right) d(m-1)+1$ linear forms to obtain the assertion of Corollary 2.10 for $k$. Consequently, $\ell_{k}=\ell_{k-1}+\left(\begin{array}{c}k-1 \\ q-1\end{array}\right) d(m-1)+1$ and $\ell_{k}$ is of the form (4), which completes the proof.

Corollary 2.12. Let $V \subset \mathbb{C}^{m}$ be an algebraic cone of pure dimension $q$ and degree $d$. There are homogeneous polynomials $f_{1}, \ldots, f_{m-q} \in \mathbb{C}\left[x_{1}, \ldots, x_{m}\right]$ such that $\operatorname{deg} f_{j} \leqslant d$ for $1 \leqslant j \leqslant m-q$, the algebraic cone $W=V\left(f_{1}, \ldots, f_{m-q}\right)$ has pure dimension $q$ and degree not exceeding $d^{m-q}$, and $V$ is the union of some irreducible components of $W$. Moreover, the matrix

$$
J\left(f_{1}, \ldots, f_{m-q}\right):=\left[\frac{\partial f_{j}}{\partial x_{k}}\right] \underset{\substack{1 \leqslant j \leqslant m-q \\ 1 \leqslant k \leqslant m}}{ }
$$

has rank $m-q$ on a Zariski open and dense subset of $W$.

Proof. If $q=m$ then the assertion is trivial. Assume that $q<m$. By the Sadullaev theorem (see [15, p. 389]) for $k=q+1$ the set $S_{m-k}(V)$ of Sadullaev's spaces of dimension $m-k$ for $V$ (i.e., linear spaces $Y \subset \mathbb{C}^{m}$ of dimension $m-k$ such that $V \subset\{x+y: x \in X, y \in Y,|y| \leqslant C(1+|x|)\}$ for some $C>0$, where $X$ is a linear complement of $Y$ ), is an open and dense subset of the Grassmann space $G_{m-k}\left(\mathbb{C}^{m}\right)$ (of $m-k$-dimensional linear subspaces of $\mathbb{C}^{m}$ ) with complement being a proper algebraic set. These are exactly the spaces $Y \in G_{m-k}\left(\mathbb{C}^{m}\right)$ for which $V \cap Y=\{0\}$. So, for any $Y \in S_{m-k}(V)$ and its linear complement $X$, the restriction to $V$ of the projection $\pi_{Y}: \mathbb{C}^{n}=X+Y \ni x+y \mapsto x \in X$ is a proper mapping. By Chevalley's theorem (see [15, p. 395]), $\pi_{Y}(V) \subset X$ is a proper algebraic set and obviously it is an algebraic cone. Moreover, from [16, Theorem 3.13] we easily deduce that $\pi_{Y}(V)$ has pure dimension $q=\operatorname{dim} X-1$, and by Fact 1.2, $\delta\left(\pi_{Y}(V)\right) \leqslant d$. So, there exists a homogeneous polynomial without multiple factors $f_{Y}$ of degree not exceeding $d$ such that $\pi_{Y}(V)+Y=V\left(f_{Y}\right)$. Consequently, $f_{Y}(x+y)=f_{Y}(x)$ for $x \in X$ and $y \in Y$ and $f_{Y}$ vanishes on $V$.

Since $V \cap Y=\{0\}$ for $Y \in S_{m-k}(V)$, by Corollary 2.10 (for $k=n$ ) and Remark 2.1 (or Corollary 2.8) we easily see that there exists a system of coordinates $x_{1}, \ldots, x_{m}$ of $\mathbb{C}^{m}$ such that for

$$
\begin{aligned}
Y_{j} & =\left\{\left(x_{1}, \ldots, x_{m}\right) \in \mathbb{C}^{m}: x_{s}=0 \text { for } j \leqslant s \leqslant j+q\right\}, \\
X_{j} & =\left\{\left(x_{1}, \ldots, x_{m}\right) \in \mathbb{C}^{n}: x_{s}=0 \text { for } 1 \leqslant s \leqslant j-1 \text { and for } j+q+1 \leqslant s \leqslant m\right\},
\end{aligned}
$$


$1 \leqslant j \leqslant m-q$, we have $Y_{j} \in S_{m-k}(V), X_{j}$ is a linear complement of $Y_{j}$ in $\mathbb{C}^{m}$. Moreover, one can assume that

$$
Z_{j}=\left\{\left(x_{1}, \ldots, x_{m}\right) \in X_{j}: x_{s}=0 \text { for } s \neq j\right\}
$$

is a Sadullaev space of $\pi_{Y_{j}}(V) \subset X_{j}$ for $1 \leqslant j \leqslant m-q$. Consequently, the polynomials $f_{Y_{j}}$ are of the forms

$$
\begin{aligned}
f_{Y_{j}}\left(x_{j}, \ldots, x_{j+q}\right)=f_{0, Y_{j}}\left(x_{j+1}, \ldots, x_{j+q}\right) x_{j}^{N_{j}}+ & f_{1, Y_{j}}\left(x_{j+1}, \ldots, x_{j+q}\right) x_{j}^{N_{j}-1} \\
& +\cdots+f_{N_{j}, Y_{j}}\left(x_{j+1}, \ldots, x_{j+q}\right)
\end{aligned}
$$

with $N_{j}>0$ and $f_{0, Y_{j}}\left(x_{j+1}, \ldots, x_{j+q}\right) \neq 0$. Moreover, for any $\left(x_{j+1}, \ldots, x_{j+q}\right) \in \mathbb{C}^{q}$ the polynomial $f_{Y_{j}}$ has a finite number of zeros $x_{j}$. So, the projection onto $\{0\} \times \mathbb{C}^{q}$ of any irreducible component of the set

$$
W=\bigcap_{1 \leqslant j \leqslant m-q}\left(\pi_{Y_{j}}(V)+Y_{j}\right)
$$

is proper and $W$ has pure dimension $q$. Since $V$ has pure dimension $q$ and $V \subset W$, we infer that $V$ is the union of some irreducible components of $W$. So, by Fact 1.2 we obtain $\operatorname{deg} V \leqslant \operatorname{deg} W \leqslant d^{m-q}$.

Since $f_{Y_{j}}$ has no multiple factors, we have $\partial f_{Y_{j}} / \partial x_{j} \neq 0$ on any irreducible component of $W$ for any $1 \leqslant j \leqslant m-q$. By the definition of $f_{Y_{j}}$ we have $\partial f_{Y_{j}} / \partial x_{k}=$ 0 in $\mathbb{C}^{n}$ for $1 \leqslant k \leqslant j-1$ and $1 \leqslant j \leqslant m-q$. So, the minor

$$
M_{0}=\operatorname{det}\left[\frac{\partial f_{Y_{j}}}{\partial x_{k}}\right]_{\substack{1 \leqslant j \leqslant m-q \\ 1 \leqslant k \leqslant m-q}}
$$

is nonzero on any irreducible component of $W$. This gives the assertion.

\section{Bertini's THEOREM}

3.1. Bertini's weak theorem. For any $a \in \mathbb{C}$, denote by $N_{a}$ the linear function $x_{1}+a x_{2}+\cdots+a^{m-1} x_{m}$. We have

Corollary 3.1 (Bertini's weak theorem). Let $C \subset \mathbb{C}^{m}$ be an algebraic cone of pure dimension $q \geqslant 1$. Then the set $A \subset \mathbb{C}$ of points $a \in \mathbb{C}$ such that $C \cap V\left(N_{a}\right)$ is an improper intersection is finite. Moreover, $\# A \leqslant d(m-q)$, where $\delta(C) \leqslant d$.

Proof. Let $k=d(m-q)$. Suppose to the contrary that $\# A>k$. Then there are $a_{1}, \ldots, a_{k+1} \in A$ such that $a_{i} \neq a_{j}$ for $1 \leqslant i, j \leqslant k+1$. Consequently, $\operatorname{dim}[C \cap$ $\left.V\left(N_{a_{j}}\right)\right]=q$ for $1 \leqslant j \leqslant k+1$. Then for any $a_{k+2}, \ldots, a_{k+q} \in \mathbb{C}$ and any $1 \leqslant i_{1}<$ $\cdots<i_{q} \leqslant d(m-q)+q$ we have $\operatorname{dim}\left[C \cap V\left(N_{a_{1}}, \ldots, N_{a_{q}}\right)\right]>0$. This contradicts Lemma 2.3 and Example 2.2 .

The properness of the intersection $C \cap V\left(N_{a}\right)$ in Corollary 3.1 cannot be replaced by transversality, as shown by the following example: 
ExAMPle 3.2. Let

$$
C=\left\{(x, y, z) \in \mathbb{C}^{3}: y^{2}-4 x z=0\right\} .
$$

Obviously $C \backslash\{0\}$ is a smooth cone of dimension 2. Let $N_{a}(x, y, z)=x+a y+a^{2} z$, $a \in \mathbb{C}$. Then $C \cap V\left(N_{a}\right) \neq \emptyset$ for any $a \in \mathbb{C}$. Moreover, if $(0,0,0) \neq\left(x_{0}, y_{0}, z_{0}\right) \in$ $C \cap V\left(N_{a}\right)$ then $z_{0} \neq 0, a=\frac{-y_{0}}{2 z_{0}}$ and $x_{0}=\frac{y_{0}^{2}}{4 z_{0}}$. So,

$$
N_{a}(x, y, z)=x+\frac{-y_{0}}{2 z_{0}} y+\frac{y_{0}^{2}}{4 z_{0}^{2}} z=\frac{-1}{4 z_{0}}\left(-4 z_{0} x+2 y_{0} y-4 x_{0} z\right)
$$

and $T_{\left(x_{0}, y_{0}, z_{0}\right)} C=V\left(N_{a}\right)$. This shows that the intersection $C \cap V\left(N_{a}\right)$ is not transversal at $\left(x_{0}, y_{0}, z_{0}\right)$.

3.2. Effective Bertini theorem. In this section we will prove some effective version of Bertini's theorem. Let us start with a lemma.

Lemma 3.3. Let $V \subset \mathbb{C}^{m}$ be an irreducible algebraic cone of dimension $q>0$ and degree $d>0$. Assume that $V \backslash\{0\}$ is smooth. Let

$$
C=\left\{N \in \mathbb{L}(m, 1): \exists_{x \in V \backslash\{0\}} N(x)=0 \wedge T_{x}(V) \subset V(N)\right\} .
$$

Then $\bar{C}$ is an irreducible algebraic cone in $\mathbb{C}^{m}$ with $\operatorname{dim} \bar{C}<m$ and

$$
\operatorname{deg} \bar{C} \leqslant 2 d^{m-q}[(m-q)(d-1)+1]^{q-1} .
$$

Proof. The inequality $\operatorname{dim} \bar{C}<m$ follows immediately from the fact that for any $x \in V \backslash\{0\}$ the set of $N \in \mathbb{L}(m, 1)$ such that $N(x)=0$ and $T_{x}(V) \subset V(N)$ is a linear space of dimension $m-q$ and that $T_{\lambda x}(V)=T_{x}(V)$ for $\lambda \in \mathbb{C} \backslash\{0\}$. Since the set $V \backslash\{0\}$ is smooth and connected, the tangent bundle

$$
W=\left\{(x, y) \in(V \backslash\{0\}) \times \mathbb{C}^{n}: y \in T_{x}(V)\right\}
$$

of $V \backslash\{0\}$ is a smooth and connected manifold, so the set $\{(x, N) \in(V \backslash\{0\}) \times$ $\left.\mathbb{L}(m, 1): T_{x}(V) \subset V(N)\right\}$ is also a smooth and connected manifold. In particular, the closure of this set is an irreducible algebraic set. Consequently, $\bar{C}$ is irreducible, as the projection onto $\mathbb{L}(m, 1)$ of the above set.

We will prove (7) by induction on $m$. For $m=1$ the assertion is trivial. Assume that it holds for $m-1$. Without loss of generality, we can assume that $V \not \subset\left\{\left(x_{1}, \ldots, x_{m}\right) \in \mathbb{C}^{m}: x_{1}=0\right\}$. By Corollary 2.12 there are nonzero homogeneous polynomials $f_{1}, \ldots, f_{m-q} \in \mathbb{C}[x]$ such that $\operatorname{deg} f_{j} \leqslant d$ for $1 \leqslant j \leqslant m-q$, the algebraic cone $V_{1}=V\left(f_{1}, \ldots, f_{m-q}\right)$ has pure dimension $q$ and $\operatorname{deg} V_{1} \leqslant d^{m-q}$, and $V$ is an irreducible component of $V_{1}$. Moreover, the matrix

$$
J\left(f_{1}, \ldots, f_{m-q}\right):=\left[\frac{\partial f_{j}}{\partial x_{k}}\right] \underbrace{}_{\substack{1 \leqslant j \leqslant m-q \\ 1 \leqslant k \leqslant m}}
$$


has rank $m-q$ on a Zariski open and dense subset of $V_{1}$. Let $b_{j, k}=\frac{\partial f_{j}}{\partial x_{k}}$ for $1 \leqslant j \leqslant m-q, 1 \leqslant k \leqslant m$ and $b_{m-q+1, k}=a_{k}$ for $1 \leqslant k \leqslant m$, where $a_{k} \in \mathbb{C}$, $N\left(x_{1}, \ldots, x_{m}\right)=a_{1} x_{1}+\cdots+a_{m} x_{m}$, and let $B=\left[b_{j, k}\right]_{\substack{1 \leqslant j \leqslant m-q+1 \\ 1 \leqslant k \leqslant m}}$. Let

$$
\begin{array}{r}
X=\left\{(x, N) \in \mathbb{C}^{m} \times \mathbb{L}(m, 1): x \neq 0, f_{1}(x)=\cdots=f_{m-q}(x)=0, N(x)=0,\right. \\
\operatorname{rank} B(x, N) \leqslant m-q\} .
\end{array}
$$

Then there exists an irreducible component $X_{1}$ of $\bar{X}$ such that $\bar{C}$ is equal to the closure of the projection of $X_{1}$ onto $\mathbb{L}(m, 1)$. So, by Fact 1.2 ,

$$
\operatorname{deg} \bar{C} \leqslant \operatorname{deg} X_{1} \leqslant \delta(\bar{X}) .
$$

Since $x_{1}$ does not vanish on $V$, we have $X_{1} \not \subset\{0\} \times \mathbb{C}^{m-1}$. Moreover, by the Euler formula $\frac{\partial f_{j}}{\partial x_{1}} x_{1}+\cdots+\frac{\partial f_{j}}{\partial x_{m}} x_{m}=f_{j} \operatorname{deg} f_{j}$, the set $X_{1}$ is an irreducible component of an algebraic set described by the equations $f_{1}(x)=\cdots=f_{m-q}(x)=0, N(x)=0$ and by $q-1$ minors of $B$ of size $(m-q+1) \times(m-q+1)$ and of degree $(m-q)(d-1)+1$. So, using Fact 1.2 and (8) we obtain the assertion.

Set $a b^{T}=a_{1} b_{1}+\cdots+a_{m} b_{m}$ for $a=\left(a_{1}, \ldots, a_{m}\right), b=\left(b_{1}, \ldots, b_{m}\right) \in \mathbb{C}^{m}$.

Theorem 3.4 (Bertini). Let $d, m, q$ be positive integers such that $m \geqslant q$, let

$$
\ell=2 d^{m-q}[(m-q)(d-1)+1]^{q-1}+m-1,
$$

and let $N_{j} \in \mathbb{L}(m, 1), 1 \leqslant j \leqslant \ell$, be a system of independent linear functions. Then

$$
E_{j_{1}, \ldots, j_{m-1}}=\left\{x \in \mathbb{C}^{m}: \exists_{a \in \mathbb{C}^{m} \backslash\{0\}} N_{j_{1}}(a)=\cdots=N_{j_{m-1}}(a)=0, a x^{T}=0\right\},
$$

for $1 \leqslant j_{1}<\cdots<j_{m-1} \leqslant \ell$ is a system of hyperplanes such that for any irreducible algebraic cone $V \subset \mathbb{C}^{m}$ with $\operatorname{dim} V=q$ and $\operatorname{deg} V \leqslant d$ such that $V \backslash\{0\}$ is smooth, there are $1 \leqslant j_{1}<\cdots<j_{m-1} \leqslant \ell$ such that the intersection $X=V \cap E_{j_{1}, \ldots, j_{m-1}}$ is transversal at any point $x \in X \backslash\{0\}$ and the set $X \backslash\{0\}$ is smooth. If moreover $q \geqslant 3$ then $X$ is irreducible.

Proof. The first assertion follows immediately from Corollary 2.7 and Lemma 3.3 The second assertion is immediate by arguing as in [7, proof of Theorem II.8.18].

REMARK 3.5. The assertion of Theorem 3.4 holds for the system of linear functions

$$
N_{j}\left(x_{0}, \ldots, x_{n}\right)=x_{0}+a_{j} x_{1}+\cdots+a_{j}^{n} x_{m},
$$

where $a_{j} \in \mathbb{C}, 1 \leqslant j \leqslant \ell$, are pairwise different numbers (see Corollary 2.8 and Remark 2.9).

From Theorem 3.4 we immediately obtain its version for projective varieties. 
Theorem 3.6 (Bertini). Let $d, m, q$ be positive integers such that $m \geqslant q$, let

$$
\ell=d^{m-q}[(m-q)(d-1)+1]^{q}+m,
$$

and let $N_{j} \in \mathbb{L}(m+1,1), 1 \leqslant j \leqslant \ell$, be a system of independent linear functions.

Then

$$
F_{j_{1}, \ldots, j_{m-1}}=\left\{x \in \mathbb{P}^{m}: \exists_{a \in \mathbb{P}^{m}} N_{j_{1}}(a)=\cdots=N_{j_{m-1}}(a)=0, a x^{T}=0\right\}
$$

for $1 \leqslant j_{1}<\cdots<j_{m-1} \leqslant \ell$ is a system of hyperplanes such that for any irreducible smooth algebraic set $V \subset \mathbb{P}^{m}$ with $\operatorname{dim} V=q$ and $\operatorname{deg} V \leqslant d$, there are $1 \leqslant j_{1}<$ $\cdots<j_{m} \leqslant \ell$ such that the intersection $X=V \cap F_{j_{1}, \ldots, j_{m-1}}$ is transversal at any point $x \in X$ and the set $X$ is smooth. If moreover $q \geqslant 2$ then $X$ is irreducible.

By a similar argument to the proof of Lemma 3.3 we will obtain

Lemma 3.7. Let $V \subset \mathbb{C}^{m}$ be an irreducible algebraic cone of dimension $q>0$ and degree $d>0$. Assume that $V \backslash\{0\}$ is smooth. Let

$$
C=\left\{N \in \mathbb{L}(m, q-1): \exists_{x \in V \backslash\{0\}} N(x)=0 \wedge \operatorname{dim}\left[T_{x}(V) \cap V(N)\right]>1\right\} .
$$

Then $\bar{C}$ is an algebraic cone in $\mathbb{L}(m, q-1)$ with $\operatorname{dim} \bar{C}<m(q-1)$ and

$$
\delta(\bar{C}) \leqslant d^{m-q}[(m-q)(d-1)+q-1] .
$$

Proof. Let $\mathcal{I} \subset \mathbb{C}\left[x_{1}, \ldots, x_{m}\right]$ be the ideal of $V$. For any $f_{1}, \ldots, f_{m-q} \in \mathcal{I}$ and $N=\left(N_{1}, \ldots, N_{q-1}\right) \in \mathbb{L}(m, q-1)$, where

$$
N_{j}\left(x_{1}, \ldots, x_{m}\right)=a_{j, 1} x_{1}+\cdots+a_{j, m} x_{m},
$$

$a_{j, k} \in \mathbb{C}$ for $1 \leqslant j \leqslant q-1$ and $1 \leqslant k \leqslant m$, we put

$$
\begin{aligned}
b_{j, k} & =\frac{\partial f_{j}}{\partial x_{k}}(x) \quad \text { for } 1 \leqslant j \leqslant m-q, \quad 1 \leqslant k \leqslant m, \\
b_{n-q+j, k} & =a_{j, k} \quad \text { for } 1 \leqslant j \leqslant q-1, \quad 1 \leqslant k \leqslant m .
\end{aligned}
$$

Let

$$
B\left(x, f_{1}, \ldots, f_{m-q}, N\right)=\left[b_{j, k}\right]_{\substack{1 \leqslant j \leqslant m-1 \\ 1 \leqslant k \leqslant m}}
$$

Then

$$
\begin{aligned}
C=\{N \in \mathbb{L}(m, q-1) & : \exists_{x \in V \backslash\{0\}} N(x)=0 \\
& \left.\wedge \forall_{f_{1}, \ldots, f_{m-q} \in \mathcal{I}} \wedge \operatorname{rank} B\left(x, f_{1}, \ldots, f_{m-q}, N\right)<m-1\right\} .
\end{aligned}
$$

Take any $x=\left(x_{1}, \ldots, x_{m}\right) \in V \backslash\{0\}$. After a linear change of coordinates, one can assume that $T_{x}(V)=\mathbb{C}^{q} \times\{0\} \subset \mathbb{C}^{m}$ and $x_{1} \neq 0$. So, for $N=\left(N_{1}, \ldots, N_{q-1}\right) \in$ $\mathbb{L}(m, q-1)$ of the form (10) we have $N(x)=0$ and $\operatorname{dim}\left[T_{x}(V) \cap V(N)\right]>1$ if and only if $N(x)=0$ and

$$
\operatorname{det}\left[a_{j, i}\right]_{\substack{1 \leqslant j \leqslant q-1 \\ 2 \leqslant i \leqslant q}}=0
$$


where we have used the Euler formula (by an analogous argument to the proof of Lemma 3.3). Consequently, the set of $N \in \mathbb{L}(m, q-1)$ such that $\operatorname{dim}\left[T_{x}(V) \cap\right.$ $V(N)]>1$ and $N(x)=0$ is an irreducible algebraic set of dimension $m(q-1)-q$. So, the fibers of the mapping

$$
W \ni(x, N) \mapsto x \in V \backslash\{0\}
$$

are irreducible sets, where

$W=\left\{(x, N) \in \mathbb{C}^{m} \times \mathbb{L}(m, q-1): x \in V \backslash\{0\}, N(x)=0, \operatorname{dim}\left[T_{x}(V) \cap V(N)\right]>1\right\}$.

Since $V \backslash\{0\}$ is a smooth set, we easily deduce that $\pi$ is a locally trivial fibration. Thus $\bar{W}$ is an irreducible algebraic set, and hence so is $\bar{C}$ (as the projection of $\bar{W}$ onto $\mathbb{L}(m, q-1))$.

The inequality $\operatorname{dim} \bar{C}<m(q-1)$ follows immediately from the fact that for any $x \in V \backslash\{0\}$ the set of $N \in \mathbb{L}(m, q-1)$ such that $\operatorname{dim}\left[T_{x}(V) \cap V(N)\right]>1$ and $N(x)=0$ is an algebraic set of dimension $m(q-1)-q$, because $\operatorname{dim} V=q$ and $T_{\lambda x}(V) \cap V(N)=T_{x}(V) \cap V(N)$ for $\lambda \in \mathbb{C} \backslash\{0\}$.

We will prove (9) by induction on $m$. For $m=1$ the assertion is trivial. Assume that it holds for $m-1$. Without loss of generality, we can assume that $V \not \subset\left\{\left(x_{1}, \ldots, x_{m}\right) \in \mathbb{C}^{m}: x_{1}=0\right\}$. By Corollary 2.12 there are nonzero homogeneous polynomials $f_{1}, \ldots, f_{m-q} \in \mathcal{I}$ such that $\operatorname{deg} f_{j} \leqslant d$ for $1 \leqslant j \leqslant m-q$, the algebraic cone $V_{1}=V\left(f_{1}, \ldots, f_{m-q}\right)$ has pure dimension $q$ and degree $\leqslant d^{m-q}$, and $V$ is an irreducible component of $V_{1}$. Moreover, the matrix

$$
J\left(f_{1}, \ldots, f_{m-q}\right):=\left[\frac{\partial f_{j}}{\partial x_{k}}\right]_{\substack{1 \leqslant j \leqslant m-q \\ 1 \leqslant k \leqslant m}}
$$

has rank $m-q$ on a Zariski open and dense subset of $V_{1}$. Let

$$
\begin{aligned}
X=\left\{(x, N) \in \mathbb{C}^{m} \times \mathbb{L}(m, q-1): f_{1}(x)\right. & =\cdots=f_{m-q}(x)=0, N(x)=0, \\
& \left.\operatorname{rank} B\left(x, f_{1}, \ldots, f_{m-q}, N\right)<m-1\right\}
\end{aligned}
$$

and let $Y \subset \mathbb{L}(m, 1)$ be the closure of the projection of $X$ onto $\mathbb{L}(m, s)$. Then $\bar{C}$ is an irreducible component of $Y$.

Since $x_{1}$ does not vanish on $V$, by the Euler formula the set $X$ can be described by $q-1$ minors of $B$ of size $(m-1) \times(m-1)$ and of degree $(m-q)(d-1)+q-1$ and by the equations $f_{1}(x)=\cdots=f_{m-q}(x)=0, N(x)=0$. So, using Fact 1.2 we obtain the assertion.

From Corollary 2.7 and Lemma 3.7 we immediately obtain 
Theorem 3.8 (Bertini). Let $d, m, q, s$ be positive integers such that $m \geqslant q$ and $q-1 \geqslant s$, let

$$
\ell=d^{m-q}[(m-q)(d-1)+q-1]+m(q-1)-1,
$$

and let $N_{j} \in \mathbb{L}(m(q-1), 1), 1 \leqslant j \leqslant \ell$, be a system of independent linear functions. Then

$$
\begin{aligned}
& K_{s, j_{1}, \ldots, j_{m(q-1)-1}}=\left\{x \in \mathbb{C}^{m}: \exists_{a=\left(a_{1}, \ldots, a_{q-1}\right) \in\left(\mathbb{C}^{m}\right)^{q-1} \backslash\{0\}} N_{j_{1}}(a)=\cdots\right. \\
& \left.\quad=N_{j_{m(q-1)-1}}(a)=0, a_{1} x^{T}=\cdots=a_{s} x^{T}=0\right\} \quad \text { for } 1 \leqslant j_{1}<\cdots<j_{m-1} \leqslant \ell
\end{aligned}
$$

is a system of linear subspaces of dimension $m-s$ such that for any irreducible algebraic cone $V \subset \mathbb{C}^{m}$ with $\operatorname{dim} V=q$ and $\operatorname{deg} V \leqslant d$ such that $V \backslash\{0\}$ is smooth, there are $1 \leqslant j_{1}<\cdots<j_{m(q-1)-1} \leqslant \ell$ such that the intersection $X=$ $V \cap V\left(K_{s, j_{1}, \ldots, j_{m(q-1)-1}}\right)$ is transversal at any point $x \in X \backslash\{0\}$ and the set $X \backslash\{0\}$ is smooth. If moreover $q \geqslant s+2$ then $X$ is irreducible.

REMARK 3.9. The estimate of the number of linear functions $\ell$ in Theorem 3.8 is better than the one obtained by repeated use of the estimate of $\ell$ in Theorem 3.4 .

REMARK 3.10. It is easy to state the above theorem for sets in projective spaces.

From Theorem 3.8 we immediately obtain (cf. Corollary 3.8)

Corollary 3.11. Under the notations and assumptions of Theorem [3.8, for any algebraic set $V=V\left(f_{1}, \ldots, f_{r}\right) \subset \mathbb{C}^{m}$ of pure dimension q, where $f_{j} \in \mathbb{C}[x]$, $\operatorname{deg} f_{j} \leqslant d$ for $1 \leqslant j \leqslant r$, we have

$$
\operatorname{deg}_{0} V=\max _{1 \leqslant j_{1}<\cdots<j_{m(q-1)-1} \leqslant \ell} \operatorname{deg}_{0}\left(V \cap K_{s, j_{1}, \ldots, j_{m(q-1)-1}}\right)
$$

for any $1 \leqslant s \leqslant q$.

\section{A Formula for the MUltiplicity of A Finite MAPPing AT ZERO}

Let $f=\left(f_{1}, \ldots, f_{m}\right):\left(\mathbb{C}^{n}, 0\right) \rightarrow\left(\mathbb{C}^{m}, 0\right)$ be a finite mapping. Then obviously $m \geqslant n$. By the multiplicity of $f$ at 0 we mean the improper intersection multiplicity

$$
i\left(\operatorname{graph} f \cdot\left(\mathbb{C}^{n} \times\{0\}\right) ;(0,0)\right)
$$

of the graph $f$ and $\mathbb{C}^{n} \times\{0\}$ at $(0,0) \in \mathbb{C}^{n} \times \mathbb{C}^{m}$ (see [1], [23]) and we denote it by $i_{0}(f)$. Note that $i_{0}(f)=\infty$ iff $f$ is not finite. If $m=n$ then $i_{0}(f)=\mu_{0}(f)$, where $\mu_{0}(f)$ denotes the covering multiplicity of $f$ at 0 , or equivalently the codimension of the ideal $\left(f_{1}, \ldots, f_{n}\right)$ in the ring $\mathcal{O}$ of germs of holomorphic functions at $0 \in \mathbb{C}^{n}$. More precisely,

$$
i_{0}(f)=\mu_{0}(f)=\operatorname{dim}_{\mathbb{C}} \mathcal{O} /\left(f_{1}, \ldots, f_{n}\right) .
$$


In the case $m>n$, we have

$$
i_{0}(f) \geqslant \operatorname{dim}_{\mathbb{C}} \mathcal{O} /\left(f_{1}, \ldots, f_{m}\right) .
$$

We will prove the following effective formula for the multiplicity $i_{0}(f)$ for finite polynomial mappings $f=\left(f_{1}, \ldots, f_{m}\right): \mathbb{C}^{n} \rightarrow \mathbb{C}^{m}$.

TheOREM 4.1. Let $\ell=d^{n}(m-n)+n$. For any independent system

$$
L_{j} \in \mathbb{L}(m, 1), \quad 1 \leqslant j \leqslant \ell,
$$

and any polynomial mapping $f: \mathbb{C}^{n} \rightarrow \mathbb{C}^{m}$ of degree $d$, finite at 0 ,

$$
i_{0}(f)=\min _{1 \leqslant i_{1}<\cdots<i_{n} \leqslant \ell} \operatorname{dim}_{\mathbb{C}} \mathcal{O} /\left(L_{i_{1}} \circ f, \ldots, L_{i_{n}} \circ f\right) .
$$

From [1, Theorem 4.4], analogously to [22, Theorem 1.1] we get

Proposition 4.2. Let $f:\left(\mathbb{C}^{n}, 0\right) \rightarrow\left(\mathbb{C}^{m}, 0\right)$ be a finite mapping and let $C_{0}$ be the tangent cone to the set $f(U)$ for a sufficiently small neighbourhood $U \subset \mathbb{C}^{n}$ of the origin. Then for any linear mapping $L \in \mathbb{L}(m, n)$ we have $i_{0}(f) \leqslant i_{0}(L \circ f)$, and equality holds if and only if $V(L) \cap C_{0}=\{0\}$.

Proof of Theorem 4.1. Since $f$ is a polynomial mapping finite at 0 , we have $\operatorname{dim} \overline{f\left(\mathbb{C}^{n}\right)}=$ $n$ and by Fact 1.1, $\operatorname{dim}\left(C_{0}\left(\overline{f\left(\mathbb{C}^{n}\right)}\right)\right)=n$. From the definition of $d$, Fact 1.3 gives $\delta\left(C_{0}\left(\overline{f\left(\mathbb{C}^{n}\right)}\right)\right) \leqslant d^{n}$. So, by Lemma 2.3 there are $1 \leqslant i_{1}, \ldots, i_{n} \leqslant \ell$ such that $V\left(L_{i_{1}}, \ldots, L_{i_{n}}\right) \cap C_{0}\left(\overline{f\left(\mathbb{C}^{n}\right)}\right)=\{0\}$. Combining (11) and Proposition 4.2 gives the assertion.

By an analogous argument to the proof of Theorem 4.1 we obtain

Corollary 4.3. Let $\ell=d(m-n)+n$. For any independent system

$$
L_{j} \in \mathbb{L}(m, 1), \quad 1 \leqslant j \leqslant \ell,
$$

and any polynomial mapping $f: \mathbb{C}^{n} \rightarrow \mathbb{C}^{m}$ with $\operatorname{deg}_{0} \overline{f\left(\mathbb{C}^{n}\right)}=d$, finite at 0 ,

$$
i_{0}(f)=\min _{1 \leqslant i_{1}<\cdots<i_{n} \leqslant \ell} \operatorname{dim}_{\mathbb{C}} \mathcal{O} /\left(L_{i_{1}} \circ f, \ldots, L_{i_{n}} \circ f\right) .
$$

From Bezout's theorem and Theorem 4.1 we obtain

Corollary 4.4. Let $f: \mathbb{C}^{n} \rightarrow \mathbb{C}^{m}$ be a polynomial mapping of degree $d$ such that $f(0)=0$. Let $m \geqslant n>q \geqslant 0, A=d^{n}(m-n)+n, B=d^{n}(n-q)+q$, and let

$$
\begin{aligned}
& L_{i}^{q} \in \mathbb{L}(m+q, 1), \quad 1 \leqslant i \leqslant A, \\
& M_{j}^{q} \in \mathbb{L}(n, 1), \quad 1 \leqslant j \leqslant B,
\end{aligned}
$$

be systems of independent functions. Then

$$
\min _{\substack{1 \leqslant i_{1}<\cdots<i_{n} \leqslant A \\ 1 \leqslant j_{1}<\cdots<j_{q} \leqslant B}} \operatorname{dim}_{\mathbb{C}} \mathcal{O} /\left(L_{i_{1}} \circ\left(f, M_{j_{1}}, \ldots, M_{j_{q}}\right), \ldots, L_{i_{n}} \circ\left(f, M_{j_{1}}, \ldots, M_{j_{q}}\right)\right)>d^{n}
$$


if and only if $\operatorname{dim}_{0} V(f) \geqslant q+1$,

REMARK 4.5. The above corollary gives an algorithm for computing $\operatorname{dim}_{0} V(f)$ and deciding whether the mapping $f$ is finite.

\section{5. ŁOJASIEWICZ EXPONENT}

The local Eojasiewicz exponent of a mapping $f:\left(\mathbb{C}^{n}, 0\right) \rightarrow\left(\mathbb{C}^{m}, 0\right)$, denoted by $\mathcal{L}_{0}(f)$, is defined to be the infimum of the set of all exponents $\nu \geqslant 1$ such that

$$
|f(z)| \geqslant C|z|^{\nu}
$$

for some constant $C>0$ in a neighbourhood of the origin in $\mathbb{C}^{n}$ (see [24]). In [12] it was proved that $\mathcal{L}_{0}(f)$ is a rational number and the infimum in the definition is in fact a minimum, provided $f$ is finite at 0 .

A. Płoski [18 gave a formula for $\mathcal{L}_{0}(f)$ for a finite mapping $f$ in terms of the characteristic polynomial, provided $n=m$. In fact, the characteristic polynomial is a polynomial with holomorphic coefficients. In the case $n=m=2$ such a formula was obtained by J. Chądzyński and T. Krasiński [2] in terms of resultants. In Proposition 5.1 below, we quote a version of Płoski's formula obtained by T. Rodak and S. Spodzieja [19, Proposition 3].

Let $P(y, t)=\sum_{j=0}^{\infty} P_{j}(y) t^{j} \in \mathbb{C}\{y, t\}$ be a power series regular of order $r+1$ in $t$. Write

$$
\Delta(P)=\min _{j=0}^{r} \frac{\operatorname{ord} P_{j}}{r+1-j} .
$$

Let $f:\left(\mathbb{C}^{n}, 0\right) \rightarrow\left(\mathbb{C}^{n}, 0\right)$ be finite at 0 . Then for every $k=1, \ldots, n$ there exists a power series $P_{k}(y, t) \in \mathbb{C}\{y, t\}$ such that for some arbitrarily small neighbourhoods $U_{0}$ and $W_{0}$ of $0 \in \mathbb{C}^{n}$ and $0 \in \mathbb{C}^{n+1}$ respectively, we have

$$
\left\{(y, t) \in W_{0}: P_{k}(y, t)=0\right\}=\left\{\left(f(z), z_{k}\right) \in \mathbb{C}^{n} \times \mathbb{C}: z \in U_{0}\right\} .
$$

Proposition 5.1. Under the above assumptions and notation,

$$
\mathcal{L}_{0}(f)=\max _{k=1}^{n} \frac{1}{\Delta\left(P_{k}\right)} .
$$

The purpose of this section is to provide an effective version of the above proposition for polynomial mappings $f:\left(\mathbb{C}^{n}, 0\right) \rightarrow\left(\mathbb{C}^{m}, 0\right), m \geqslant n$, finite at 0 (see Theorem 5.7 below). Let us start with some supporting facts.

From [22, Theorem 2.1 and its proof] we have the following proposition.

Proposition 5.2. Let $f:\left(\mathbb{C}^{n}, 0\right) \rightarrow\left(\mathbb{C}^{m}, 0\right)$ be a finite mapping and let $C_{0}$ be the tangent cone to the set $f(U)$ for a sufficiently small neighbourhood $U \subset \mathbb{C}^{n}$ of the origin. Then for any linear mapping $L \in \mathbb{L}(m, n)$ we have $\mathcal{L}_{0}(f) \leqslant \mathcal{L}_{0}(L \circ f)$, and equality holds if and only if $V(L) \cap C_{0}=\{0\}$. 
From Proposition 5.2. Facts 1.1, 1.3 and Lemma 2.3 we immediately obtain

Corollary 5.3. Let $m \geqslant n$ be positive integers, let $\ell=d^{n}(m-n)+n$ and let $L_{j} \in \mathbb{L}(m, 1), 1 \leqslant j \leqslant \ell$, be a system of independent linear functions. Then for any polynomial mapping $f:\left(\mathbb{C}^{n}, 0\right) \rightarrow\left(\mathbb{C}^{m}, 0\right)$ finite at 0 with $\operatorname{deg} f \leqslant d$ we have

$$
\mathcal{L}_{0}(f)=\min _{1 \leqslant i_{1}<\cdots<i_{n} \leqslant \ell} \mathcal{L}_{0}\left(\left(L_{i_{1}}, \ldots, L_{i_{n}}\right) \circ f\right) .
$$

We will need the following two propositions, proved by A. Płoski [17], [18, Proposition 1.3, Theorem 3.5] for $m=n$ and generalized by S. Spodzieja 22., Corollary 1.3, Proposition 3.1] to $m>n$.

Proposition 5.4. Let $f:\left(\mathbb{C}^{n}, 0\right) \rightarrow\left(\mathbb{C}^{m}, 0\right)$ be a holomorphic mapping finite at 0 . If $g:\left(\mathbb{C}^{n}, 0\right) \rightarrow\left(\mathbb{C}^{m}, 0\right)$ is a holomorphic mapping such that $\operatorname{ord}(f-g)>\mathcal{L}_{0}(f)$ then $g$ is finite at 0 and $\mathcal{L}_{0}(g)=\mathcal{L}_{0}(f)$.

Proposition 5.5. Let $f: \mathbb{C}^{n} \rightarrow \mathbb{C}^{m}$ be a polynomial mapping finite at 0 with $\operatorname{deg} f \leqslant d$. Then $\mathcal{L}_{0}(f) \leqslant d^{n}$.

We will consider the Łojasiewicz exponent of polynomial mappings. Let $f=$ $\left(f_{1}, \ldots, f_{m}\right): \mathbb{C}^{n} \rightarrow \mathbb{C}^{m}, m \geqslant n$, be a polynomial mapping such that $f(0)=0$. Let $d$ be the degree of $f$. The basic difficulty in obtaining effective formulas for the Łojasiewicz exponent is to determine the characteristic polynomials $P_{1}, \ldots, P_{n}$ with holomorphic coefficients. To bypass this difficulty and get the usual polynomials, we will reduce the problem to finding the exponent for proper mappings. For this purpose we define a mapping $H_{L}: \mathbb{C}^{n} \rightarrow \mathbb{C}^{n}$ by

$$
H_{f, L}(z)=L \circ f(z)+\left(z_{1}^{d^{n}+1}, \ldots, z_{n}^{d^{n}+1}\right), \quad z=\left(z_{1}, \ldots, z_{n}\right) \in \mathbb{C}^{n},
$$

where $d \geqslant \operatorname{deg} f$ and $L \in \mathbb{L}(m, n)$. Obviously $H_{L}$ is a proper mapping.

From Corollary 5.3 and Propositions 5.4, 5.5] we obtain

Corollary 5.6. Let $m \geqslant n$ be positive integers, let $\ell=d^{n}(m-n)+n$, and let $L_{j} \in \mathbb{L}(m, 1), 1 \leqslant j \leqslant \ell$, be a system of independent linear functions. Then for any polynomial mapping $f:\left(\mathbb{C}^{n}, 0\right) \rightarrow\left(\mathbb{C}^{m}, 0\right)$ finite at 0 with $\operatorname{deg} f \leqslant d$ we have

$$
\mathcal{L}_{0}(f)=\min _{1 \leqslant i_{1}<\cdots<i_{n} \leqslant \ell} \mathcal{L}_{0}\left(H_{f,\left(L_{i_{1}}, \ldots, L_{i_{n}}\right)}\right) .
$$

Set

$$
\mathbb{M}(m, n)=\mathbb{L}(m, n) \times \mathbb{L}(n, 1)
$$

and let $\Phi_{f, L, N}: \mathbb{C}^{n} \rightarrow \mathbb{C}^{n+1}$, where $(L, N) \in \mathbb{M}(m, n)$, be given by

$$
\Phi_{f, L, N}(z)=\left(H_{f, L}(z), N(z)\right) .
$$


The mapping $\Phi_{f, L, N}$ is proper and consequently $\Phi_{f, L, N}\left(\mathbb{C}^{n}\right) \subset \mathbb{C}^{n+1}$ is an algebraic set of pure dimension $n$. Hence, there exists a polynomial $P_{f, L, N} \in \mathbb{C}[y, t]$, where $y=\left(y_{1}, \ldots, y_{n}\right)$ and $y_{1}, \ldots, y_{n}, t$ are independent variables, of the form

$$
P_{f, L, N}(, y, t)=\sum_{j=0}^{r_{f, L, N}} P_{f, L, N, j}(y) t^{j}
$$

such that

$$
\Phi_{f, L, N}\left(\mathbb{C}^{n}\right)=V\left(P_{f, L, N}\right) .
$$

Since $H_{f, L, N}$ is a proper mapping, we may assume that $P_{f, L, N, r_{f, L, N}} \neq 0$. Moreover, we may assume that $P_{f, L, N}$ is irreducible.

To use the Płoski formula $(\mathbb{P})$, we have to guarantee the fulfillment of assumptions (12). This can be done by selecting sufficiently many independent linear functions of $L \in \mathbb{L}(m, 1)$ and $N \in \mathbb{L}(n, 1)$. Namely, let

$$
\ell_{N}=n+\left[\left(d^{n}+1\right)^{n}-1\right] n(n-1), \quad \ell_{L}=d^{n}(m-n)+n .
$$

Take any families of independent linear functions

$$
N_{i} \in \mathbb{L}(n, 1), \quad 1 \leqslant i \leqslant \ell_{N}, \quad L_{s} \in \mathbb{L}(m, 1), \quad 1 \leqslant s \leqslant \ell_{L} .
$$

Let

$$
\mathbb{I}_{L}=\left\{\mathbf{s}=\left(s_{1}, \ldots, s_{n}\right) \in \mathbb{N}^{n}: 1 \leqslant s_{1}<\cdots<s_{n} \leqslant \ell_{L}\right\} .
$$

Obviously $\# \mathbb{I}_{L}=\left(\begin{array}{c}\ell_{L} \\ n\end{array}\right)$. Set

$$
L_{\mathbf{s}}=\left(L_{s_{1}}, \ldots, L_{s_{n}}\right) \in \mathbb{L}(m, n) \text { for } \mathbf{s} \in \mathbb{I}_{L} .
$$

For any $1 \leqslant i \leqslant \ell_{N}$ and $\mathbf{s} \in \mathbb{I}_{L}$ we define a mapping $\Phi_{f,(i, \mathbf{s})}: \mathbb{C}^{n} \rightarrow \mathbb{C}^{n} \times \mathbb{C}$ by

$$
\Phi_{f,(i, \mathbf{s})}(z)=\Phi_{f, L_{\mathbf{s}}, N_{i}}
$$

i.e., $\Phi_{f,(i, \mathbf{s})}=\left(H_{f, \mathbf{s}}(z), N_{i}(z)\right)$, where $H_{f, \mathbf{s}}(z)=L_{\mathbf{s}}(f(z)(z))+\left(z_{1}^{d^{n}+1}, \ldots, z_{n}^{d^{n}+1}\right)$.

The main result of this section is the following theorem.

TheOREM 5.7. For any polynomial mapping $f:\left(\mathbb{C}^{n}, 0\right) \rightarrow\left(\mathbb{C}^{m}, 0\right)$ finite at 0 of degree $d$ the Lojasiewicz exponent $\mathcal{L}_{0}(f)$ is given by

$$
\mathcal{L}_{0}(f)=\min _{\mathbf{s} \in \mathbb{I}_{L}} \max _{1 \leqslant i \leqslant \ell_{N}} \frac{1}{\Delta\left(P_{f, L_{\mathbf{s}}, N_{i}}\right)} .
$$

We will precede the proof of the above theorem with a remark and an example.

Remark 5.8. Since $P_{f, L, N}$ vanishes exactly on the image of $\Phi_{f, L, N}$, it can be effectively computed, for instance by using Gröbner bases (see [6]). One can also compute it as a multipolynomial resultant of the coordinates of $\Phi_{f, L, N}$ (see [5]). An important point in this construction is properness of the mappings $H_{f, L, N}$ and $\Phi_{f, L, N}$, which we owe to the component $\left(z_{1}^{d^{n}+1}, \ldots, z_{n}^{d^{n}+1}\right)$. Therefore, the polynomials $P_{f, L, N}$ in Theorem 5.7 are regular in $t$ and we can compute the numbers 
$\Delta\left(P_{f, L, N}\right)$. If we omit this component, with fixed $L, M$ and $N=z_{k}$ the polynomials $P_{f, L, N}$ may not satisfy (12) (see Example 5.9 below).

Example 5.9. The polynomial mapping $f=\left(f_{1}, f_{2}\right): \mathbb{C}^{2} \rightarrow \mathbb{C}^{2}$ defined by

$$
f_{1}\left(z_{1}, z_{2}\right)=z_{1}\left(1-z_{1}^{2}-z_{2}^{2}\right), \quad f_{2}\left(z_{1}, z_{2}\right)=z_{2}\left(1-z_{1}^{2}-z_{2}^{2}\right)
$$

is finite at $(0,0) \in \mathbb{C}^{2}$ and it is not proper. For the irreducible polynomial

$$
P\left(y_{1}, y_{2}, t\right)=t^{3}\left(y_{1}^{2}+y_{2}^{2}\right)-t y_{1}^{2}+y_{1}^{3}
$$

we have $P\left(f\left(z_{1}, z_{2}\right), z_{1}\right)=0$ for $\left(z_{1}, z_{2}\right) \in \mathbb{C}^{2}$. Moreover, the polynomial $P$ is not regular in $t$ and we cannot compute the number $\Delta(P)$.

Proof of Theorem 5.7. By an analogous argument to the proof of [19, Lemma 1] we obtain

Lemma 5.10. If $P, Q, R \in \mathbb{C}\{y, t\}$ are series such that

$$
P(y, t)=\sum_{j=0}^{\infty} P_{j}(y) t^{j}, \quad Q(y, t)=\sum_{j=0}^{\infty} Q_{j}(y) t^{j},
$$

and $Q=P R$ and for some $r \geqslant 0$ we have ord $P_{j}$, ord $Q_{j}>0, j=0, \ldots, r$, then

$$
\min _{0 \leqslant j \leqslant r} \frac{\operatorname{ord} P_{j}}{r+1-j} \leqslant \min _{0 \leqslant j \leqslant r} \frac{\operatorname{ord} Q_{j}}{r+1-j} .
$$

If additionally ord $R=0$, then equality holds in (15).

Fix $\mathbf{s}=\left(s_{1}, \ldots, s_{n}\right)$ with $1 \leqslant s_{1}<\cdots<s_{n} \leqslant \ell_{L}$ and let $L_{\mathbf{s}}=\left(L_{s_{1}}, \ldots, L_{s_{n}}\right) \in$ $\mathbb{L}(m, n)$.

LEMmA 5.11. There are $1 \leqslant i_{1}<\cdots<i_{n} \leqslant \ell_{N}$ such that

$$
V\left(H_{f, L_{\mathbf{s}}}\right) \cap V\left(N_{i_{j}}\right)=\{0\} \quad \text { for } 1 \leqslant j \leqslant n .
$$

Proof. Since $H_{L, M}$ has no zeros at infinity, it is proper. Consequently, by Bezout's theorem it has at most $\left(d^{n}+1\right)^{n}-1$ zeros in $\mathbb{C}^{n} \backslash\{0\}$. Thus by Corollary 2.10 we obtain (16).

Lemma 5.12. If $N \in \mathbb{L}(n, 1)$ satisfies (16), then for any sufficiently small $\epsilon>0$ the germs of the sets

$$
A_{\epsilon}=\left\{\left(H_{f, L_{\mathbf{s}}}(z), N(z)\right):|z|<\epsilon\right\} \quad \text { and } \quad B=\left\{(y, t): P_{f, L_{\mathbf{s}}, N}(y, t)=0\right\}
$$

at $0 \in \mathbb{C}^{n+1}$ are equal.

Proof. It suffices to prove that for any $\epsilon>0$ there exists a neighbourhood $U_{0}$ of $0 \in \mathbb{C}^{n+1}$ such that $A_{\epsilon} \cap U_{0}=B \cap U_{0}$. Indeed, suppose there exists $\epsilon>0$ such that for any neighbourhood $U_{0}$ of zero we have $A_{\epsilon} \cap U_{0} \neq B \cap U_{0}$. Obviously $A_{\epsilon} \subset B$, hence $A_{\epsilon} \cap U_{0} \subset B \cap U_{0}$. So, the inclusion $A_{\epsilon} \cap U_{0} \supset B \cap U_{0}$ fails, i.e., there exists 
a sequence $\left(y_{\nu}, t_{\nu}\right) \in B \cap U_{0}$ such that $\left(y_{\nu}, t_{\nu}\right) \rightarrow(0,0)$ and $\left(y_{\nu}, t_{\nu}\right) \notin A_{\epsilon} \cap U_{0}$. By (14) there exists a sequence $z_{\nu} \in \mathbb{C}^{n}$ such that $y_{\nu}=H_{L_{\mathbf{s}}}\left(z_{\nu}\right)$ and $t_{\nu}=N\left(z_{\nu}\right)$. Thus

$$
\left(H_{f, L_{\mathbf{s}}}\left(z_{\nu}\right), N\left(z_{\nu}\right)\right) \rightarrow(0,0)
$$

as $\nu \rightarrow \infty$. The sequence $\left(z_{\nu}\right)$ is bounded (by properness of $H_{f, L_{\mathbf{s}}}$ ); we can assume that $z_{\nu} \rightarrow z_{0}$ as $\nu \rightarrow \infty$. Then

$$
\left(H_{f, L_{\mathbf{s}}}\left(z_{0}\right), N\left(z_{0}\right)\right)=(0,0)
$$

so, by (16), $z_{0}=0$ and $z_{\nu} \rightarrow 0$ as $\nu \rightarrow \infty$. On the other hand, $\left(y_{\nu}, t_{\nu}\right) \notin A_{\epsilon} \cap U_{0}$ implies $\left|z_{\nu}\right| \geqslant \epsilon$, a contradiction.

By Lemmas 5.11 and [5.12, for any fixed $\mathbf{s}$ we can find $1 \leqslant i_{1}, \ldots, i_{n} \leqslant \ell_{N}$ such that $P_{f, L_{\mathbf{s}}, N_{i_{j}}}$ satisfy the assumption (12) of Proposition 5.1. Note that if $P_{f, L_{\mathbf{s}}, N_{i}}$ does not satisfy (12), the characteristic polynomial for $\Phi_{f, L_{\mathbf{s}}, N_{i}}$ is a divisor of $P_{f, L_{\mathrm{s}}, N_{i}}$. Hence, in view of Lemma 5.10, we obtain

$$
\mathcal{L}_{0}\left(H_{f, L_{\mathbf{s}}}\right)=\max _{1 \leqslant i \leqslant \ell_{N}} \frac{1}{\Delta\left(P_{f, L_{\mathbf{s}}, N_{i}}\right)} .
$$

This together with Corollary 5.6 gives the assertion of Theorem 5.7 .

Theorem 5.7 simplifies in the case of proper polynomial mappings finite at 0 . By an analogous argument to that in the proof of Theorem 5.7 we obtain

Corollary 5.13. Let $d, n, m, m \geqslant n$, be positive integers, let

$$
\ell_{N}=\left(d^{n}-1\right) n(n-1)+n, \quad \ell_{L}=d^{n}(m-n)+n,
$$

and let $N_{i} \in \mathbb{L}(n, 1), 1 \leqslant i \leqslant \ell_{N}, L_{j} \in \mathbb{L}(m, 1), 1 \leqslant j \leqslant \ell_{L}$, be sequences of independent functions. Then for any proper polynomial mapping $f:\left(\mathbb{C}^{n}, 0\right) \rightarrow$ $\left(\mathbb{C}^{n}, 0\right)$ finite at 0 of degree $d$ there exist polynomials

$$
P_{f,\left(L_{s_{1}}, \ldots, L_{s_{n}}\right), N_{i}} \in \mathbb{C}[y, t], \quad 1 \leqslant i \leqslant \ell_{N}, \quad 1 \leqslant s_{1}<\cdots<s_{n} \leqslant \ell_{L},
$$

regular in $t$, such that the set $V\left(P_{f,\left(L_{s_{1}}, \ldots, L_{s_{n}}\right), N_{i}}\right)$ is equal to the image of $\mathbb{C}^{n}$ under the mapping $z \mapsto\left(\left(L_{s_{1}}, \ldots, L_{s_{n}}\right) \circ f(z), N_{i}(z)\right)$ and

$$
\mathcal{L}_{0}(f)=\min _{1 \leqslant s_{1}<\cdots<s_{n} \leqslant \ell_{L}} \max _{1 \leqslant i \leqslant \ell_{N}} \frac{1}{\Delta\left(P_{f,\left(L_{s_{1}}, \ldots, L_{s_{n}}\right), N_{i}}\right)} .
$$

Theorem 5.7 and Corollary 5.13 give effective algorithms for computing the multiplicity and the local Łojasiewicz exponent of a polynomial mapping $\mathbb{C}^{n} \rightarrow \mathbb{C}^{n}$ finite at 0 . Moreover, they allow one to decide whether a polynomial mapping $\left(\mathbb{C}^{n}, 0\right) \rightarrow\left(\mathbb{C}^{n}, 0\right)$ is finite at 0 . Namely, by calculating the Lojasiewicz exponents of mappings $(f, M): \mathbb{C}^{n} \rightarrow \mathbb{C}^{m+q}$ for $M \in \mathbb{L}(n, q)$ instead of $f$, we obtain 
Proposition 5.14. Let $d, n, m, m \geqslant n$, be positive integers, let $0 \leqslant q \leqslant n$, and let

$$
\ell_{L}=d^{n}(m-n)+n, \quad \ell_{M}=d^{n}(n-q)+q .
$$

Let $L_{j} \in \mathbb{L}(m, 1), 1 \leqslant j \leqslant \ell_{L}, M_{k} \in \mathbb{L}(n, 1), 1 \leqslant k \leqslant \ell_{M}$, be sequences of independent functions. Let $\left.f:\left(\mathbb{C}^{n}, 0\right) \rightarrow \mathbb{C}^{m}, 0\right)$ be a polynomial mapping of degree d. Set

$$
H_{f, L_{\mathbf{s}}, M_{\mathbf{k}}}(z)=L_{\mathbf{s}} \circ\left(f(z), M_{\mathbf{k}}(z)\right)+\left(z_{1}^{d^{n}+1}, \ldots, z_{n}^{d^{n}+1}\right)
$$

for $L_{\mathbf{s}}=\left(L_{s_{1}}, \ldots, L_{s_{n}}\right), \mathbf{s}=\left(s_{1}, \ldots, s_{n}\right) \in \mathbb{I}_{L}$ and $M_{\mathbf{k}}=\left(M_{k_{1}}, \ldots, M_{k_{q}}\right), \mathbf{k}=$ $\left(k_{1}, \ldots, k_{q}\right), 1 \leqslant k_{1}<\cdots<k_{q} \leqslant \ell_{M}$.

1. If $\operatorname{dim}_{0} V(f) \geqslant q+1$ then

$$
\min _{\mathbf{s}, \mathbf{k}} \mathcal{L}_{0}\left(H_{f, L_{\mathbf{s}}, M_{\mathbf{k}}}\right) \geqslant d^{n}+1 .
$$

2. If $\operatorname{dim}_{0} V(f) \leqslant q$ then

$$
\min _{\mathbf{s}, \mathbf{k}} \mathcal{L}_{0}\left(H_{f, L_{\mathbf{s}}, M_{\mathbf{k}}}\right) \leqslant d^{n} .
$$

\section{REFERENCES}

[1] R. Achilles, P. Tworzewski, T. Winiarski, On improper isolated intersection in complex analytic geometry. Ann. Polon. Math. 51 (1990), 21-36.

[2] J. Chądzyński, T. Krasiński, Resultant and the Eojasiewicz exponent. Ann. Polon. Math. 61 (1995), 95-100.

[3] R.N. Draper, Intersection theory in analytic geometry. Math. Ann. 180 (1969), 175-204.

[4] W. Fulton, Intersection theory. Springer, Berlin, 1984.

[5] I.M. Gelfand, M.M. Kapranov, A.V. Zelevinsky, Discriminants, resultants, and multidimensional determinants. Reprint of the 1994 edition, Modern Birkhäuser Classics, Birkhäuser Boston, Boston, MA, 2008.

[6] M.-G. Gruel, G. Pfister, A SINGULAR introduction to commutative algebra. Springer, Berlin, 2008.

[7] R. Hartshorne, Algebraic geometry. Springer, 1977.

[8] S.L. Kleiman, Bertini and his two fundamental theorems. Studies in the history of modern mathematics, III. Rend. Circ. Mat. Palermo (2) Suppl. No. 55 (1998), 9-37.

[9] K. Kurdyka, B. Osińska-Ulrych, G. Skalski, S. Spodzieja, Effective Eojasiewicz gradient inequality for generic Nash functions with isolated singularity. Bull. Soc. Sci. Lett. Łódź Sér. Rech. Déform. 66 (2016), 47-64.

[10] K. Kurdyka, S. Spodzieja, Separation of real algebraic sets and the Eojasiewicz exponent. Proc. Amer. Math. Soc. 142 (2014), 3089-3102.

[11] K. Kurdyka, S. Spodzieja, A. Szlachcińska, Metric properties of semialgebraic sets. Discrete Comput. Geom. 55 (2016), 786-800.

[12] M. Lejeune-Jalabert, B. Teissier, Clôture intégrale des idéaux et équisingularité. With an appendix by J.-J. Risler. Ann. Fac. Sci. Toulouse Math. (6) 17 (2008), 781-859.

[13] S. Łojasiewicz, Ensembles semi-analytiques. Preprint IHES, 1965.

[14] S. Lojasiewicz, Sur les trajectoires du gradient d'une fonction analytique. In: Geometry Seminars, 1982-1983, Univ. Stud. Bologna, Bologna, 1984, 115-117.

[15] S. Łojasiewicz, Introduction to complex analytic geometry. Birkhäuser, Basel, 1991.

[16] D. Mumford, Algebraic geometry I, Complex projective varieties. Springer, 1991.

[17] A. Płoski, Sur l'exposant d'une application analytique. II. Bull. Polish Acad. Sci. Math. 33 (1985), 123-127.

[18] A. Płoski, Multiplicity and the Eojasiewicz exponent. Banach Center Publications 20 (1988), 353-364. 
[19] T. Rodak, S. Spodzieja, Effective formulas for the local Eojasiewicz exponent. Math. Z. 268 (2011), 37-44.

[20] J-P. Serre, Local algebra. Springer Monogr. Math., Springer, Berlin, 2000.

[21] S. Spodzieja, The Eojasiewicz exponent at infinity for overdetermined polynomial mappings. Ann. Polon. Math. 78 (2002), 1--10.

[22] S. Spodzieja, Multiplicity and the Lojasiewicz exponent. Ann. Polon. Math. 73 (2000), 257267.

[23] W. Stoll, The multiplicity of a holomorphic map. Invent. Math. 2 (1966), 15--58.

[24] B. Teissier, Variétés polaires. I. Invariants polaires des singularités d'hypersurfaces. Invent. Math. 40 (1977), 267-292.

[25] H. Whitney, Tangents to an analytic variety. Ann. of Math. 81 (1965), 496-549.

(Tomasz Rodak) Faculty of Mathematics and Computer Science, University of Lodz, S. Banacha 22, 90-238 Łódź, Poland

Email address: tomasz.rodak@wmi.uni.lodz.pl

(Adam Różycki) Faculty of Mathematics and Computer Science, University of Lodz, S. Banacha 22, 90-238 Łódź, Poland.

Email address: adam.rozycki@wmi.uni.lodz.pl

(Stanisław Spodzieja) Faculty of Mathematics and Computer Science, University of Lodz, S. Banacha 22, 90-238 Łódź, Poland

Email address: stanislaw.spodzieja@wmii.uni.lodz.pl 